\title{
Effects of $p$-benzoquinone and melatonin on amyloid fibrillogenesis of hen egg-white lysozyme
}

\author{
Steven S.-S. Wang *, Po-Han Chen, Ying-Tz Hung \\ Department of Chemical Engineering, National Taiwan University, No. 1, Sec. 4, Roosevelt Road, Taipei 10617, Taiwan
}

Available online 26 July 2006

\begin{abstract}
More than 20 different human proteins can fold abnormally resulting in the formation of pathological deposits and several lethal degenerative diseases. Despite extensive investigations on amyloid fibril formation, the detailed molecular mechanism remained far from complete. In this work, utilizing hen egg-white lysozymes as a model system, two objectives were pursued: (1) to search for suitable conditions for producing amyloid fibrils and (2) to investigate inhibitory activities of two potential molecules against lysozyme fibril formation. Via numerous spectroscopic analyses and electron microscopy, our results showed that the formation of lysozyme amyloid fibrils at $\mathrm{pH} 2.0$ was considerably increased by the addition of salt. Moreover, the inhibition of lysozyme amyloid formation by either $p$-benzoquinone or melatonin followed a concentration-dependent fashion. Furthermore, $p$-benzoquinone, in comparison with melatonin, served as a more effective inhibitor against amyloid fibril formation of lysozyme. We believe that a better understanding of how hen egg-white lysozymes aggregate will not only aid in deciphering the molecular mechanism of amyloid fibrillogenesis, but also shed light on a rational design of effective therapeutics for amyloidogenic diseases.
\end{abstract}

(c) 2006 Elsevier B.V. All rights reserved.

Keywords: Lysozyme; Amyloid; Fibril; Aggregation; Inhibitor

\section{Introduction}

The amyloidoses are a group of protein misfolding or conformational diseases which arise when a constituent protein or peptide undergoes a change in size or fluctuation in shape, with resultant self-association and tissue deposition. Up to now, more than 20 different human proteins and peptides, including transthyretin, $\alpha$-synuclein, $\beta 2$-macroglobulin and $\beta$-amyloid, have been isolated as the fibrillar components of diseaseassociated amyloid deposits [12,24,25,30,48,59]. These amyloidogenic diseases individually have their unique neuropathological, clinical and biochemical characteristics and their corresponding amyloidogenic precursor proteins have unrelated functions and exhibit little sequence or structural homology. However, the molecular events underlying these disease processes appear to be the same. The mechanism involves an aberrant structural transition in a normally innocuous and functional protein $[12,24,30,48,55,59]$. Despite extensive investigations on amyloid fibril formation, the detailed molecular mechanism remained largely unknown.

\footnotetext{
* Corresponding author. Tel.: +8862 3366 5870; fax: +886223623040. E-mail address: sswang@ntu.edu.tw (S.S.-S. Wang).
}

Aggregation/fibrillization is generally considered to be associated with partially folded intermediate species that are susceptible to self-assembly owing to the exposure of hydrophobic core [37]. The pathological conformers resulted from aggregation/fibrillization process in these protein misfolding diseases are rich in the content of cross- $\beta$-pleated sheet and extensive protein fibrillogenesis or aggregation often occurs leading to an accumulation of the abnormally folded proteins that correlates with cell death. The formation of these pathological conformers is influenced by genetic mutations, protein concentration, chaperones and environmental factors [6-8,22,24,30,54].

Mounting evidence has indicated that amyloid fibril formation is not only possible to a certain group of proteins associated with amyloidogenic diseases. Proteins, for instance, glucagons, synthetic poly-L-lysine, bovine serum albumin and myoglobin that are not linked to any known amyloidogenic disease have been induced in vitro to self-assemble into amyloid fibrils under certain favorable conditions such as heating, low $\mathrm{pH}$, agitation, pressure and the presence of cosolvent $[17,29,52,53]$. The idea that the formation of fibrillar species in vitro as a generic property of peptides/proteins, even in non-disease associated ones, is gaining renewed attention $[1,4,9,12,13,23]$.

Hen egg-white lysozyme, an enzyme with 129 amino acids that lyses the cell walls of bacteria, has been extensively stud- 
ied. Structurally, the monomeric form of the lysozyme has four disulfide bonds and adopts mainly helical conformation $(\sim 30 \%$ $\alpha$-helix; $\sim 6 \% \beta$-sheet) [60]. Reports showed that, while only $\sim 40 \%$ of the enzyme is identical in sequence to the human lysozyme, which has been found to form amyloid fibrils that are responsible for hereditary non-neuropathic systemic amyloidosis [44], these two forms of proteins, nevertheless, retain a high degree of structural homology. Recent reports have found that, in addition to human lysozyme, several other variants of lysozymes are also capable of forming fibrillar species that present characteristics of amyloid fibrils produced from the group of proteins related to clinical amyloidoses [5,18-20,28].

In the current work, utilizing hen egg-white lysozymes as a model system, attempts were made not only to seek out the suitable conditions of producing amyloid fibrils, but also to investigate the anti-amyloidogenic and anti-aggregating activities of potential molecules/compounds against the formation of aggregated species or amyloid fibrils of lysozyme. Through turbidity measurement, fluorescence spectroscopy with ThT dye, circular dichroism absorption spectroscopy, Congo red binding assay and electron microscopy, we first demonstrated that the formation of hen egg-white lysozyme (HEWL) amyloid fibrils at acidic condition ( $\mathrm{pH}$ 2.0) was considerably increased by the addition of salt. Next, two potential amyloid inhibitors, $p$-benzoquinone and melatonin, against HEWL fibrillogenesis were tested in this study. Our results indicated that the inhibition of HEWL amyloid formation by either $p$-benzoquinone or melatonin followed a concentration-dependent manner. Moreover, $p$-benzoquinone served as a more effective inhibitor in comparison with melatonin against amyloid fibril formation of HEWLs. We believe that a better understanding of how hen eggwhite lysozymes self-assemble will not only aid in deciphering the molecular mechanism of amyloid fibrillogenesis, but also shed light on a rational design of effective therapeutics for amyloidogenic diseases.

\section{Materials and methods}

\subsection{Proteins and reagents}

Hen egg-white lysozyme (HEWL; EC 3.2.1.17) was purchased from Merck (Germany) and without further purification. Hydrochloric acid $(\mathrm{HCl})$ and di-potassium hydrogen phosphate $\left(\mathrm{K}_{2} \mathrm{HPO}_{4}\right)$ were obtained from Merck (Germany). Potassium dihydrogen phosphate $\left(\mathrm{KH}_{2} \mathrm{PO}_{4}\right)$ and di-sodium hydrogen phosphate $\left(\mathrm{Na}_{2} \mathrm{HPO}_{4}\right)$ were purchased from Showa Chemical, Co. Ltd. (Japan). All other chemicals, unless otherwise specified, were obtained from Sigma Chemical Co. (St. Louis, MO, USA).

\subsection{Lysozyme sample solution preparation}

Sample solutions of $2 \mathrm{mg} / \mathrm{mL}$ HEWL were prepared by dissolving $0.1 \mathrm{~g}$ lyophilized HEWL in $50 \mathrm{~mL}$ of hydrochloric acid ( $\mathrm{pH} 2.0)$ with or without salts $(136.7 \mathrm{mM} \mathrm{NaCl}, 2.68 \mathrm{mM}$ $\mathrm{KCl})$. HEWL sample solutions were first mixed via vortexing and then incubated at $55^{\circ} \mathrm{C}$ during the course of fibril formation/aggregation process.

\subsection{Turbidimetric aggregation analysis}

Absorbance at $450 \mathrm{~nm}$ was monitored at different times during the course of fibril formation/aggregation process. A $300 \mu \mathrm{L}$ of lysozyme samples taken at different times were added to a well of 96-well plate. The absorbance was measured using a $\mu$ Quant Microplate reader (Bio-Tek Instruments, VT, USA). In order to well suspend the fibrillar species, the lysozyme sample solutions were mixed by vortexing prior to each absorbance measurement. All measurements were taken in triplicate.

\subsection{Thioflavin T fluorescence (ThT) assay}

A $40 \mu \mathrm{L}$ of lysozyme samples taken at different times were mixed with $960 \mu \mathrm{L}$ of $10 \mu \mathrm{M}$ thioflavin $\mathrm{T}$ (ThT) in phosphate buffered saline (PBS) with $0.01 \%(\mathrm{w} / \mathrm{v})$ sodium azide. ThT fluorescence intensity measurements were performed by exciting samples at $440 \mathrm{~nm}$ and recording emission intensities at $485 \mathrm{~nm}$ using a F-2500 Fluorescence Spectrophotometer (Hitachi, Japan). All measurements were taken in triplicate.

\subsection{Congo red binding assay}

To assess the presence of amyloid fibrils in the calcitonin solutions, Congo red binding studies were performed. Congo red dye was dissolved in PBS to a final concentration of $112 \mu \mathrm{M}$. Congo red absorbances of lysozyme sample solutions and the free dye controls were determined by adding Congo red to a final concentration of $18 \mu \mathrm{M}$ and acquiring spectral measurements from 400 to $700 \mathrm{~nm}$ at $25^{\circ} \mathrm{C}$ using a Spectronic Genesys 5 Spectrophotometer (Spectronic Instrument, USA) [27]. Both the lysozyme solutions and the control solutions were allowed to interact with Congo red for at least $30 \mathrm{~min}$ prior to recording their spectra. All measurements were taken in triplicate.

\subsection{Circular dichroism spectroscopy}

Circular dichroism (CD) spectra of lysozyme samples were recorded on a JASCO J-715 (150-S Type) spectrometer (Sunway Scientific Corporation) at $25^{\circ} \mathrm{C}$ using a bandwidth of $2.0 \mathrm{~nm}$, a step interval of $0.1 \mathrm{~nm}$ and an averaging time of $2 \mathrm{~s}$. A $0.01 \mathrm{~cm}$ quartz cell was used for far-UV (190-260 nm) measurements. Three scans each of duplicate samples were measured and averaged. Control buffer scans were run in duplicate, averaged and then subtracted from the sample spectra. The results were plotted as ellipticity (millidegree) versus wavelength (nm).

\subsection{Transmission electron microscopy}

A $20 \mu \mathrm{L}$ sample was fixed for $5 \mathrm{~min}$ in $0.25 \%$ glutaraldehyde (Electron Microscopy Sciences, Hatfield, PA, USA) in PBS, pH 7.2. The sample was then placed on carbon stabilized, formvar coated grids. Grids were negatively stained with $2 \%$ (w/v) aqueous uranyl acetate (Electron Microscopy Sciences, Hatfield, PA, USA) and then examined and photographed in a JEOL, JEM1200EX II transmission electron microscope (Tokyo, Japan) at an accelerating voltage of $80 \mathrm{kV}$. 


\section{Results and discussion}

\subsection{Effect of salt on HEWL aggregation kinetics and amyloid fibril formation}

The absorbance or turbidity of HEWL sample solutions measured at $450 \mathrm{~nm}$ was used as an indication of the degree of aggregation. As depicted in Fig. 1A, the absorbance of HEWL in hydrochloric acid ( $\mathrm{pH}$ 2.0) with salt increased markedly during the course of aggregation. In contrast, for the case of HEWL dissolved in hydrochloric acid ( $\mathrm{pH} 2.0$ ) without salt, no detectable elevation in turbidity at $450 \mathrm{~nm}$ was observed even at 55 days after the initiation of aggregation (see Fig. 1A). Therefore, our turbidity measurements suggested that the addition of salt into hydrochloric acid at $\mathrm{pH} 2.0$ resulted in the production of larger HEWL species.

ThT is believed to interact rapidly and specifically with amyloid fibrils [32] and an increase in ThT fluorescence intensity has been reported as an important indicator of the presence of amyloid fibril [32]. As seen in Fig. 1B, in the absence of salt, no significant ThT fluorescence emission was observed over 55 days, indicating that amyloid fibrils were not produced during the course of incubation. However, when dissolved in hydrochloric acid ( $\mathrm{pH}$ 2.0) with salt, the HEWL solution was observed to be visibly turbid and exhibited a dramatic increase in ThT fluorescence intensity as amyloid fibril formation progressed. In addition, the two-phase nucleation-dependent polymerization behavior with sigmoidal time-course response was observed in HEWLs dissolved in hydrochloric acid ( $\mathrm{pH} 2.0$ ) with salt. This nucleation-dependent polymerization phenomenon is sufficiently characterized by two key parameters, the lag time of nucleation (lag) phase and the growth rate of elongation phase. The lag time and the growth rate for HEWL in hydrochloric acid without salt were determined to be 32.14 day and 0.12 day $^{-1}$, respectively, while 5.55 day and 0.26 day $^{-1}$ for the case with salt addition. It was apparent that the existence of $\mathrm{NaCl}$ and $\mathrm{KCl}$ elevated the growth rate by around two-fold and dramatically shortened the duration of lag phase.

Congo red absorption spectroscopy was then utilized to probe for the presence of cross- $\beta$-pleated sheet structure associated with amyloids. We show in Fig. 2A and B that, relative to the case in the hydrochloric acid solution alone, HEWLs solubilized in the hydrochloric acid/salt solution shifted the spectral properties of Congo red and exhibited a second shoulder peak at around $540 \mathrm{~nm}$, which is indicative of strong binding between HEWL and Congo red dye and generation of appreciable amount of amyloid fibrils (even after 52 days). Further confirmation that the salt-induced HEWL fibrillization came from electron micrographs of amyloid fibrils formed from HEWL in the absence and presence of salt in the dissolving solvent (Fig. 3).

\subsection{Effect of salt on HEWL conformation}

To elucidate the role of salt in changes in structure of HEWL, the CD spectroscopy was employed to investigate the information of structural transition, the secondary structure in particular. As can be seen in Fig. 4A, even at day 41 after the initiation
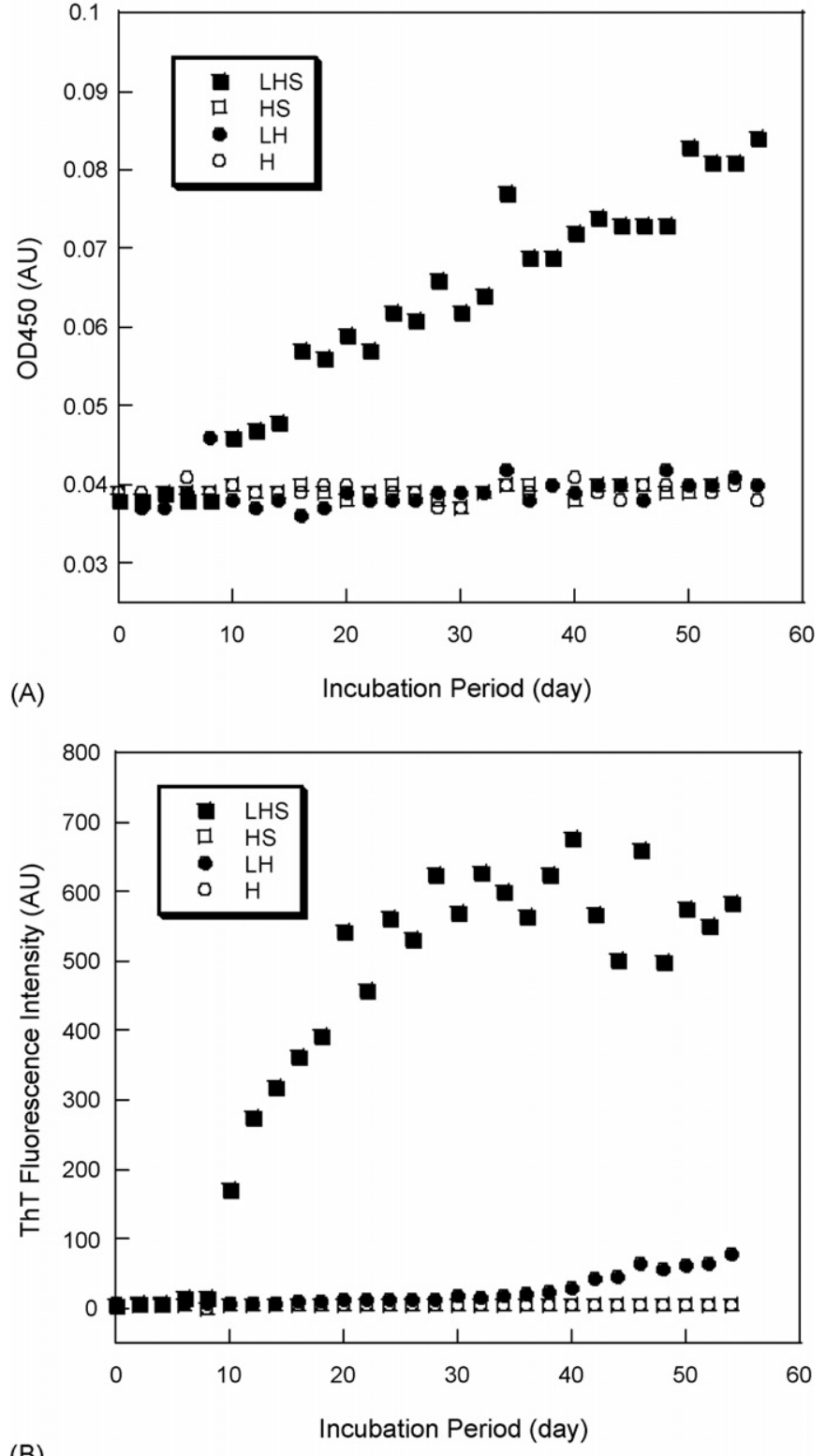

(B)

Fig. 1. The effect of salt addition on kinetics of aggregation/fibril formation of hen egg-white lysozyme. (A) The extent of aggregation was measured via turbidity as a function of incubation time of aggregation. Lysozymes were dissolved in hydrochloric acid ( $\mathrm{pH}$ 2.0) with salt (LHS) and without salt (LH). Lysozyme samples aged for different time periods were then tested for aggregation. Data represent the mean turbidity measurement of at least five independent experiments $(n \geq 5)$. (B) The extent of fibril formation was measured via ThT fluorescence as a function of incubation time of fibrillogenesis. Lysozymes were dissolved in hydrochloric acid ( $\mathrm{pH} 2.0)$ with salt (LHS) and without salt (LH). Lysozyme samples aged for different time periods were then tested for fibril formation. Data represent the mean ThT fluorescence measurement of at least five independent experiments $(n \geq 5)$.

of aggregation, the CD spectra of HEWL in hydrochloric acid ( $\mathrm{pH}$ 2.0) alone showed a secondary structure with a shoulder at $\sim 222 \mathrm{~nm}$ as well as an absorption minimum at $\sim 208 \mathrm{~nm}$, reflecting an $\alpha$-helix-rich conformation. However, the addition of salt to HEWL permitted an occurrence of structural transition, resulting in a striking alteration in the relative proportion of solution structure (Fig. 4B). The CD spectra obtained from HEWL 


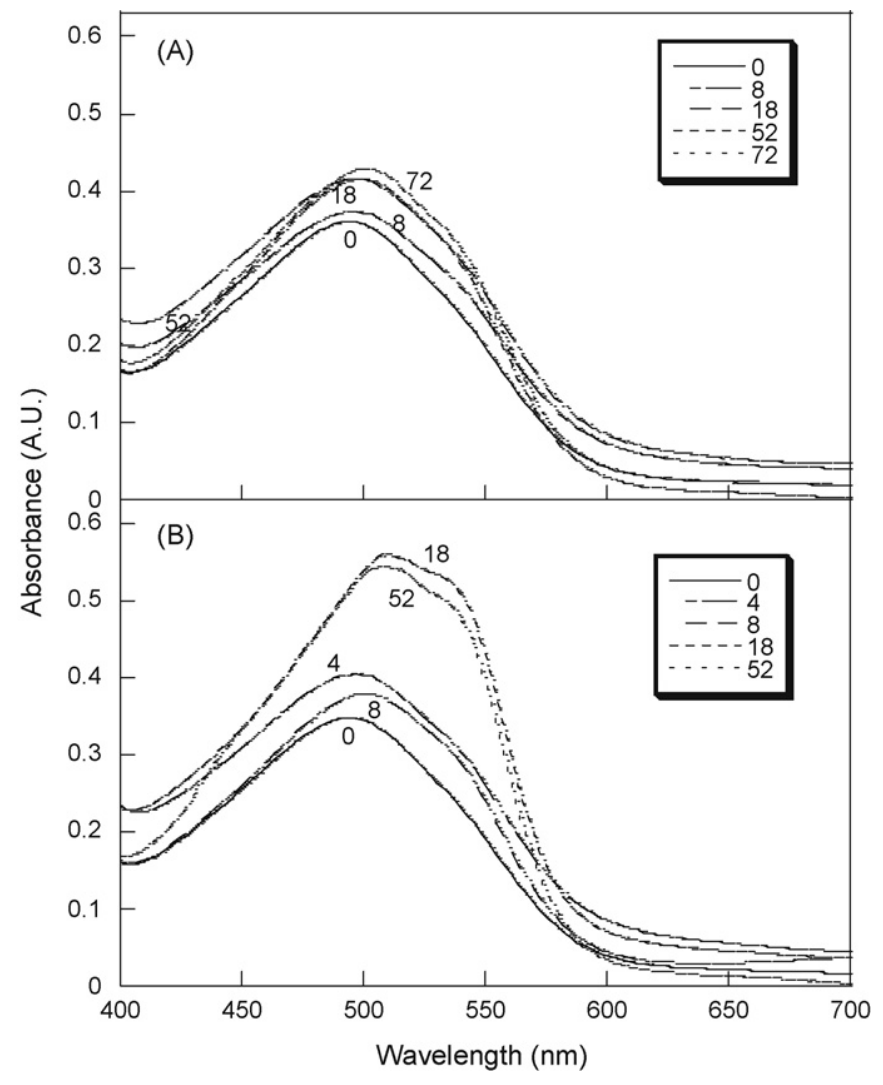

Fig. 2. (A) Representative Congo red absorption spectra of hen egg-white lysozyme dissolved in hydrochloric acid ( $\mathrm{pH} 2.0)$ without salt taken at different incubation times $(0,8,18,52$ and 72 days). (B) Representative Congo red absorption spectra of hen egg-white lysozyme dissolved in hydrochloric acid ( $\mathrm{pH} 2.0)$ with salt taken at different incubation times $(0,4,8,18$ and 52 days).

in hydrochloric acid in the presence of salt were observed to exhibit a characteristic pattern of $\beta$-sheet conformation with an absorption minimum around $216 \mathrm{~nm}$, clearly suggesting that the conformation of HEWL species in hydrochloric acid/salt was predominately $\beta$-structure. Obviously, by comparing Fig. 4A and $\mathrm{B}$, the $\alpha$ to $\beta$ transition in HEWL structure was drastically induced upon treatment of salt.

Several lines of evidence suggest that the presence of salt has an impact on the formation of amyloid species and aggregates as well as their conformational change. It was found by Wang et al. that suspending bovine calcitonin in salt solutions (e.g. $\mathrm{CaCl}_{2}$ and $\mathrm{MgCl}_{2}$ ) yielded peptide solutions with enriched $\beta$-sheet content, a critical feature/trait of amyloid proteins/peptides [62]. Report also showed that the addition of $\mathrm{NaCl}$ to HEWL-water-ethanol solution facilitated the fibrillogenesis along with gel formation [20]. Moreover, Fujiwara et al., employing various concentrations of $\mathrm{NaCl}$ along with time-resolved neutron scattering, proposed a strong correlation between the salt concentration and the structure of HEWL aggregated species [19]. They further concluded that the structural diversity of aggregated species was due to the variation in the level of electrostatic shielding resulted from salt addition [19].

Our observations are in line with aforementioned and other researches that the rate of association of amyloid proteins is highly dependent on the presence of salt, implying the pivotal
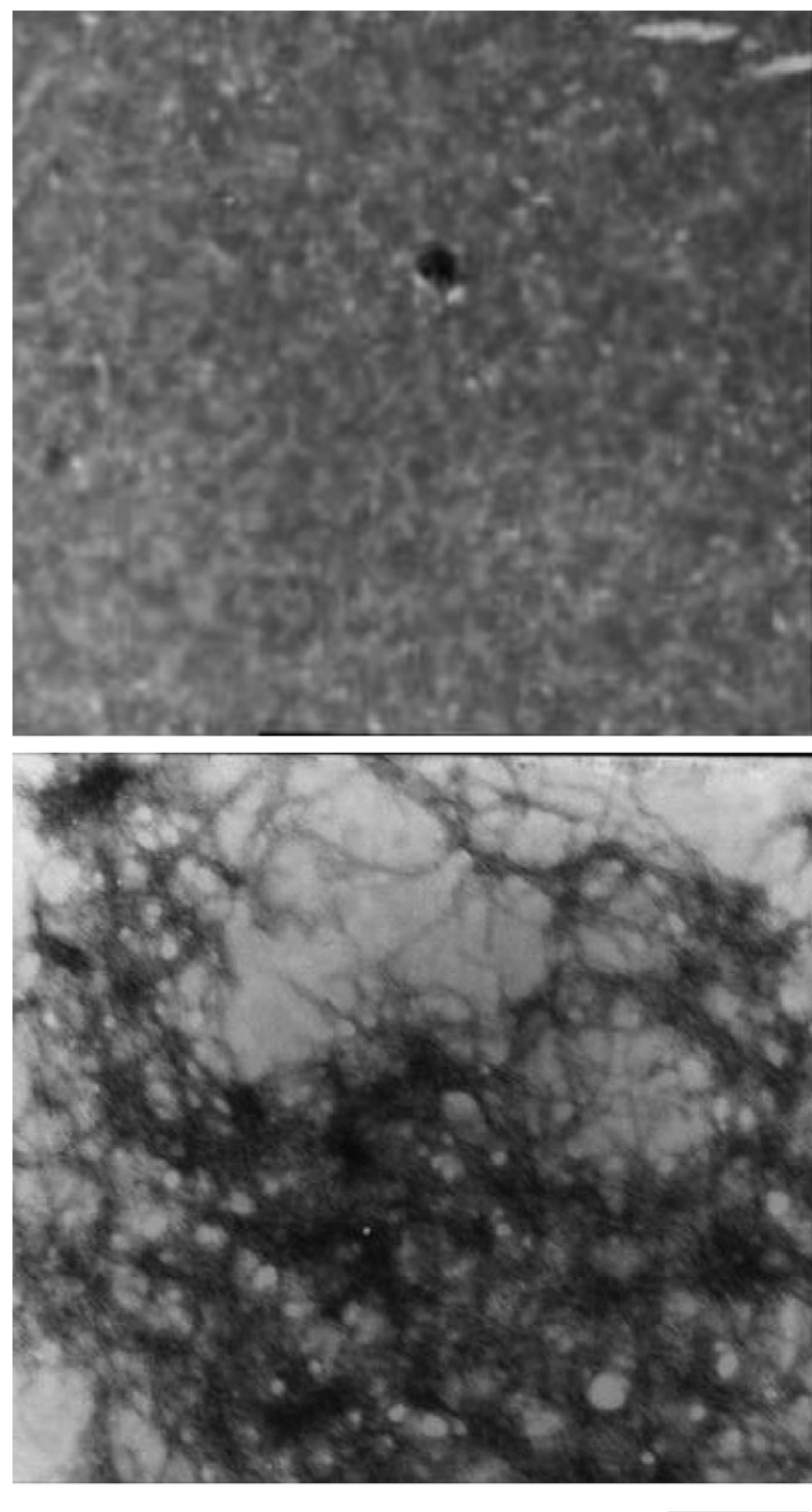

$250 \mathrm{~nm}$

Fig. 3. Electron micrographs of negatively stained hen egg-white lysozyme Lysozyme sample was prepared in hydrochloric acid without (top) and with salt (bottom) on day 52 after the initiation of aggregation.

role of electrostatic interaction in the process of HEWL fibril formation. Possible explanation is as follows: due to the deviation from the isoelectric point of HEWL ( $\mathrm{p} I=\sim 10.7$ ) [46], the experimental environment of $\mathrm{pH} 2.0$ in the absence of salt led to a surplus of charges on the surface of HEWL molecules, resulting in a lower probability of collisions between HEWLs as well as the formation of aggregates/fibrils.

\subsection{Effects of p-benzoquinone and melatonin on HEWL fibril formation}

In exploring if $p$-benzoquinone or melatonin exerted an inhibitory action against the formation of HEWL fibrils at $\mathrm{pH}$ 


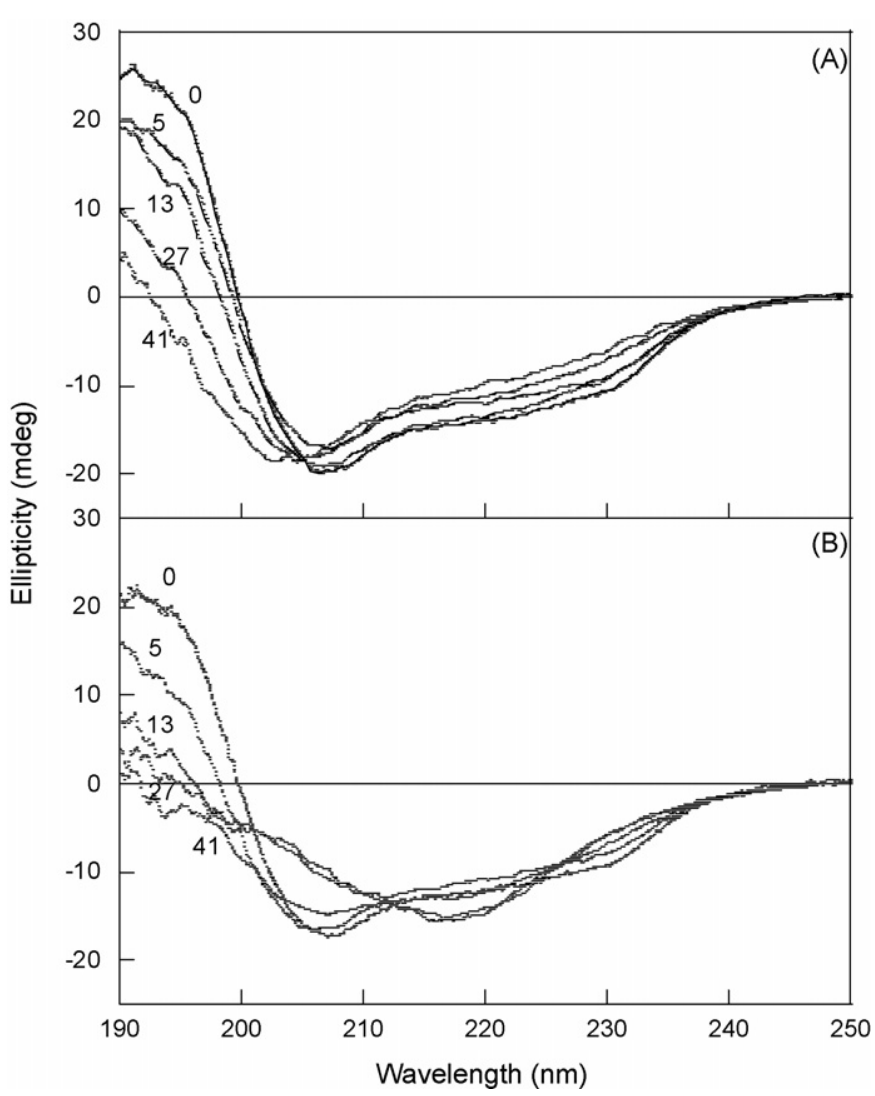

Fig. 4. (A) Representative CD spectra of hen egg-white lysozyme prepared in hydrochloric acid at $\mathrm{pH} 2.0$ without salt taken at different incubation times $(0,5$, 13, 27 and 41 days). (B) Representative CD spectra of hen egg-white lysozyme prepared in hydrochloric acid at $\mathrm{pH} 2.0$ with salt taken at different incubation times $(0,5,13,27$ and 41 days).

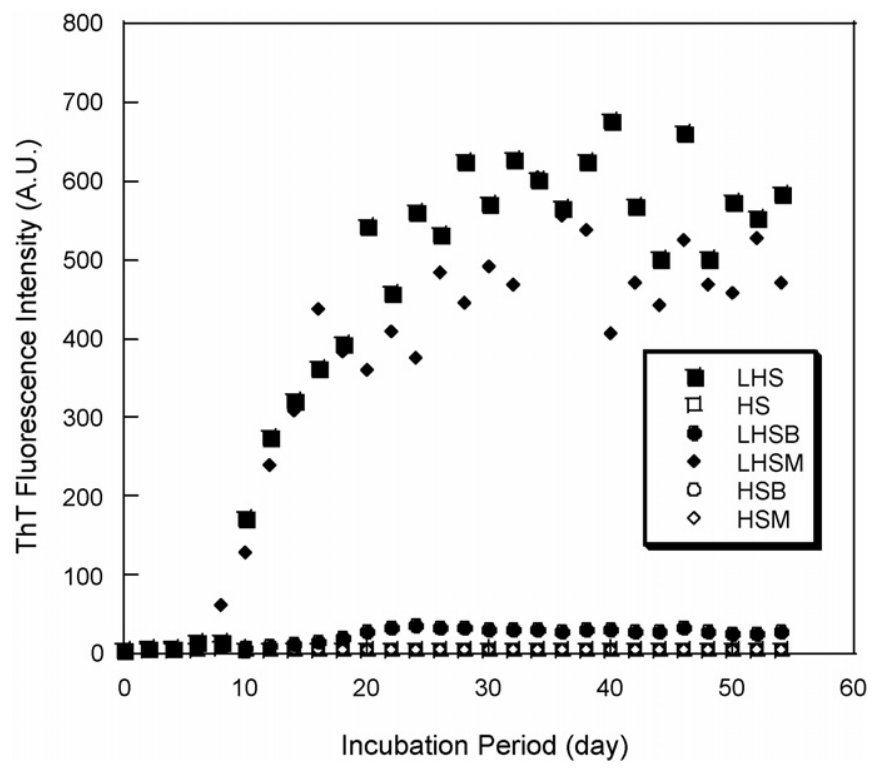

Fig. 5. The effect of $p$-benzoquinone or melatonin on the kinetics of amyloid fibril formation of hen egg-white lysozyme. The extent of fibril formation was measured via ThT fluorescence as a function of incubation time of fibril formation process. The concentrations of $p$-benzoquinone and melatonin were set at 1000 and $500 \mu \mathrm{M}$, respectively. Each point represents the average of at least five independent measurements $(n \geq 5)$.
2.0, we first monitored the changes of ThT fluorescence intensity and Congo red binding spectra of $\mathrm{HEWL}$ in $\mathrm{HCl} /$ salt with and without $500 \mu \mathrm{M}$ melatonin or $1000 \mu \mathrm{M} p$-benzoquinone as a function of incubation period. Our results showed that $p$-benzoquinone at $1000 \mu \mathrm{M}$ possessed an inhibitory potency on HEWL fibril formation in $\mathrm{HCl} / \mathrm{salt}$ solution, while this effect was not observed in the case with melatonin at the concentration of $500 \mu \mathrm{M}$. As illustrated in Fig. 5, the treatment with $p$-benzoquinone at $1000 \mu \mathrm{M}$ significantly reduced the ThT fluorescence intensity emitted by HEWL; however, there was little difference in ThT fluorescence signal observed between the HEWL solutions with or without melatonin. As a complementary measure of the presence of amyloid fibril, Congo red binding to HEWL samples was performed. Owing to the lack of shoulder peak at $\sim 540 \mathrm{~nm}$ in the Congo red absorbance spectra, no appreciable amount of amyloid fibril was observed in the HEWL sample with $1000 \mu \mathrm{M} p$ benzoquinone (Fig. 6A). In Fig. 6B, as opposed to the HEWL sample with $p$-benzoquinone, an obvious absorption shoulder peak at $\sim 540 \mathrm{~nm}$ was found in the Congo red binding spectra upon addition of melatonin, suggesting the superior anti-amyloidogenic potency was found in $p$-benzoquinone over melatonin.

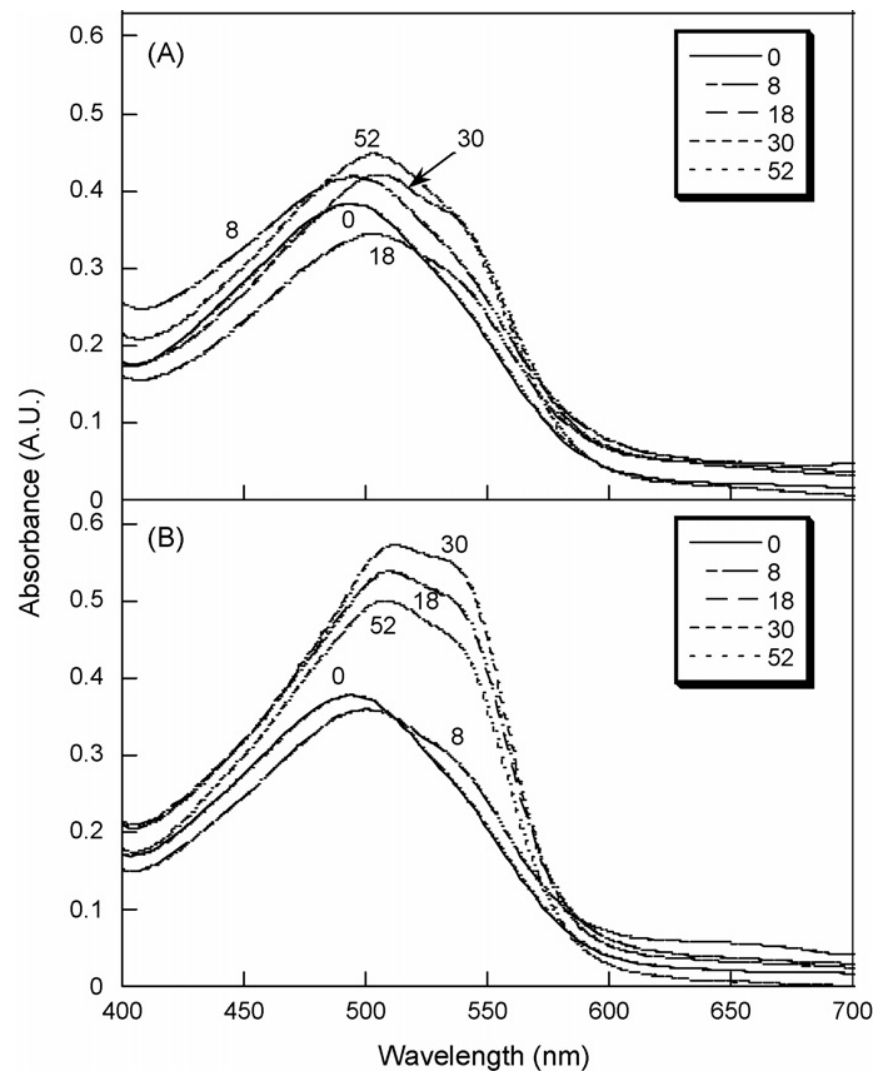

Fig. 6. (A) Representative Congo red binding spectra of hen egg-white lysozyme dissolved in hydrochloric acid/salt ( $\mathrm{pH} 2.0)$ with $1000 \mu \mathrm{M}$ of $p$-benzoquinone taken at different incubation times $(0,8,18,30$ and 52 days). (B) Representative Congo red binding spectra of hen egg-white lysozyme dissolved in hydrochloric acid/salt ( $\mathrm{pH} 2.0$ ) with $500 \mu \mathrm{M}$ of melatonin taken at different incubation times $(0,8,18,30$ and 52 days $)$. 


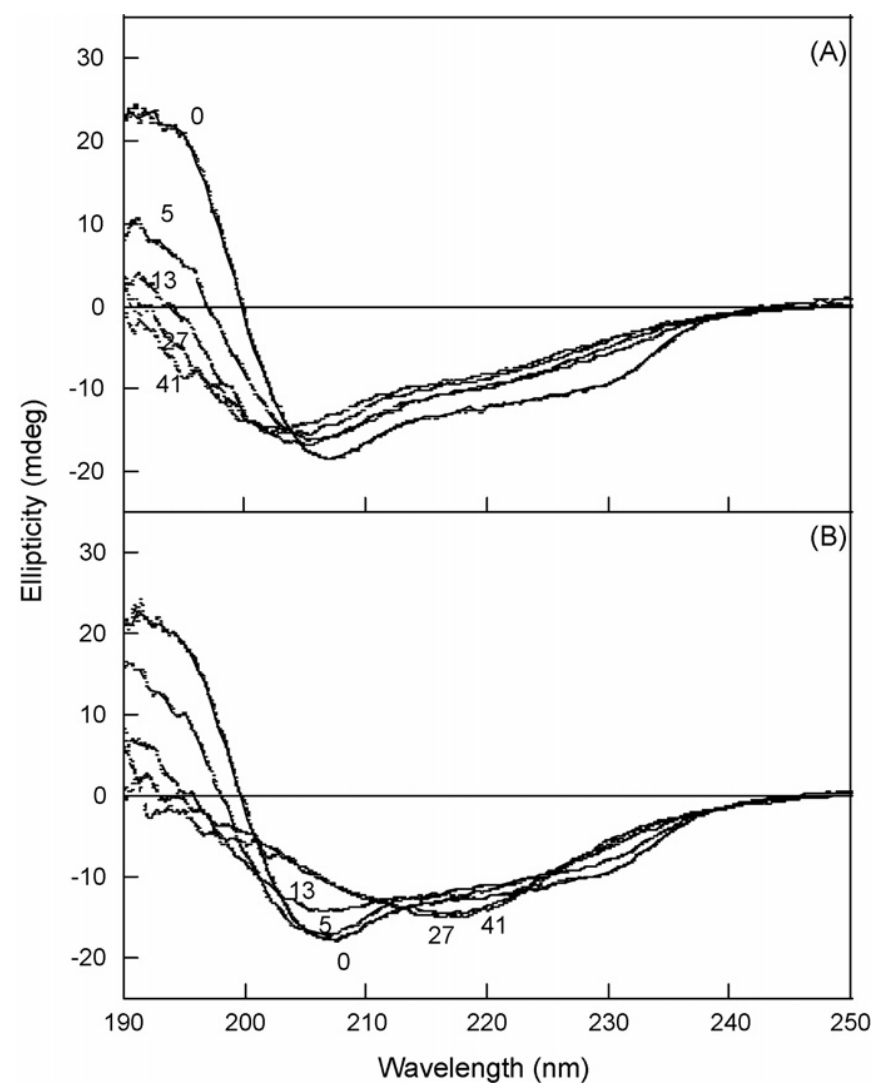

Fig. 7. (A) Representative CD spectra of hen egg-white lysozyme dissolved in hydrochloric acid/salt ( $\mathrm{pH} 2.0)$ with $1000 \mu \mathrm{M}$ of $p$-benzoquinone taken at different incubation times $(0,5,13,27$ and 41 days). (B) Representative CD spectra of hen egg-white lysozyme dissolved in hydrochloric acid/salt $(\mathrm{pH} 2.0)$ with $500 \mu \mathrm{M}$ of melatonin taken at different incubation times $(0,5,13,27$ and 41 days).

\subsection{Effects of p-benzoquinone and melatonin on HEWL conformation}

The influence of $p$-benzoquinone and melatonin on the secondary structure transitions in HEWL is illustrated in Fig. 7A and $\mathrm{B}$, respectively. The far-UV CD spectra of HEWL solutions with and without melatonin $(500 \mu \mathrm{M})$ both exhibited $\beta$-sheetrich conformation, suggesting that little or no inhibitory effect on HEWL conformation was possessed by $500 \mu \mathrm{M}$ melatonin (see Figs. 4B and 7B). However, the presence of $1000 \mu \mathrm{M} p$ benzoquinone evidently prevented a structural transition from the native $\alpha$-helix rich HEWL conformer to amyloidogenic $\beta$ sheet rich species (see Fig. 7A).

\subsection{Concentration-dependent effects of melatonin and p-benzoquinone on HEWL fibril formation}

Through ThT fluorescence measurements, we further investigated the dependence of concentration levels of $p$-benzoquinone or melatonin on the HEWL fibril generation at fibrillogenesis favorable condition ( $\mathrm{pH} 2.0$ with salt). As seen in Fig. 8A, $p$ benzoquinone retarded HEWL aggregation/fibril formation in a concentration-dependent manner. The inhibitory effect was decreased as the concentration of $p$-benzoquinone dropped from
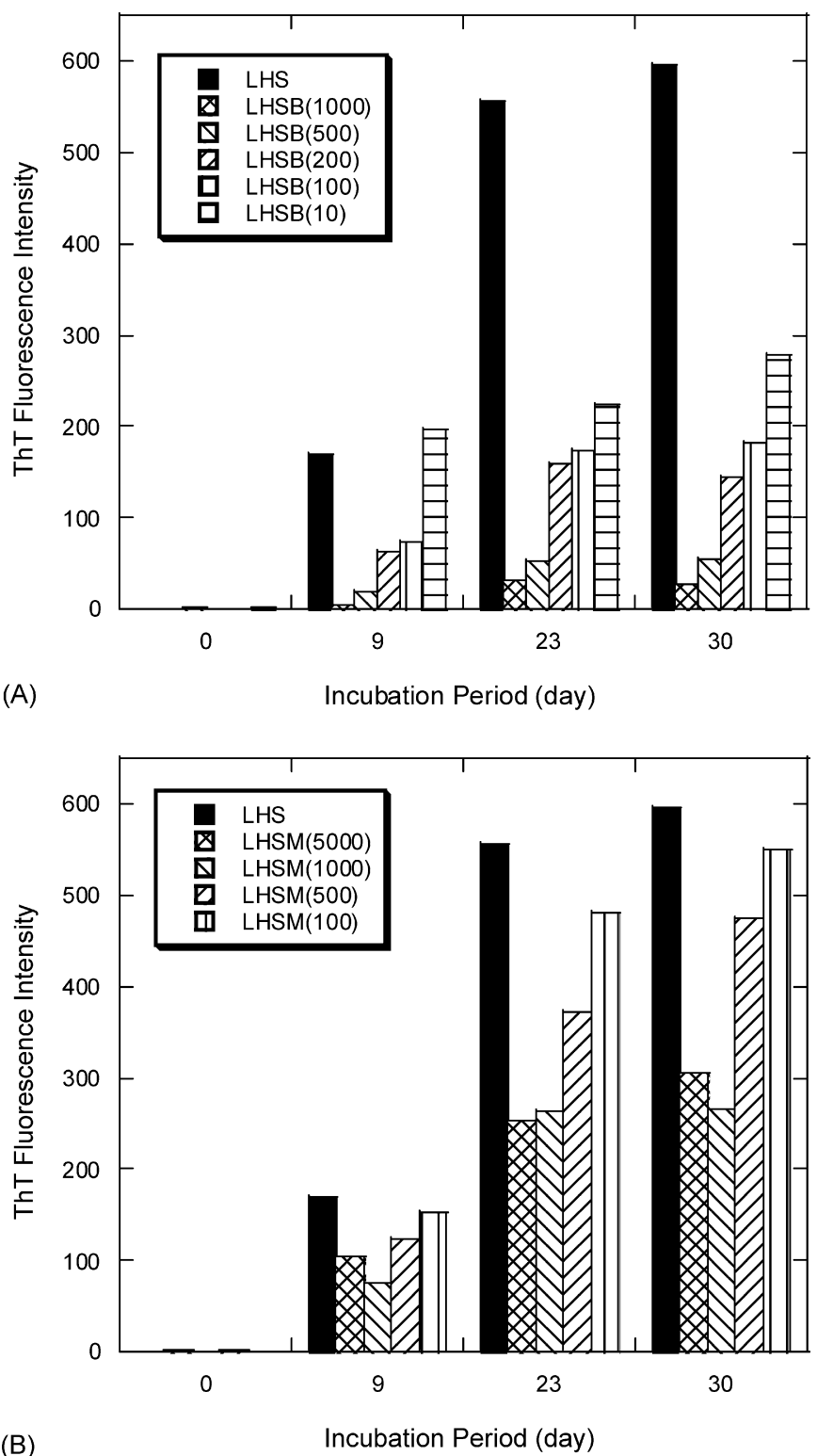

Fig. 8. The effect of dosage of (A) $p$-benzoquinone and (B) melatonin on amyloid fibril formation of hen egg-white lysozyme as monitored by ThT fluorescence. Five $(10,100,200,500$ and $1000 \mu \mathrm{M})$ and four $(100,500,1000$ and $5000 \mu \mathrm{M})$ different concentration levels are used for $p$-benzoquinone and melatonin, respectively.

1000 to $10 \mu \mathrm{M}$ and near-maximal inhibition was reached with $p$ benzoquinone at $1000 \mu \mathrm{M}(95.6 \%$ decrease of ThT fluorescence relative to that of HEWL with salt). As for melatonin, no noticeable anti-amyloidogenic action was observed below the concentration of $1000 \mu \mathrm{M}$ while marked preventive effect became discernible at concentrations above $1000 \mu \mathrm{M}$ (Fig. 8B). For instance, after 30 days of incubation, the percentage reductions of ThT fluorescence by melatonin at 500, 1000 and $5000 \mu \mathrm{M}$ were approximately $20.2 \%, 55.3 \%$ and $48.9 \%$, respectively. The best anti-amyloidogenic inhibitory effect was obtained with $1000 \mu \mathrm{M}$ melatonin. Similar conclusions can also be drawn from our Congo red binding data (data not shown).

In order to retrieve insights in regards to protein structural transition, the effects of melatonin and $p$-benzoquinone 

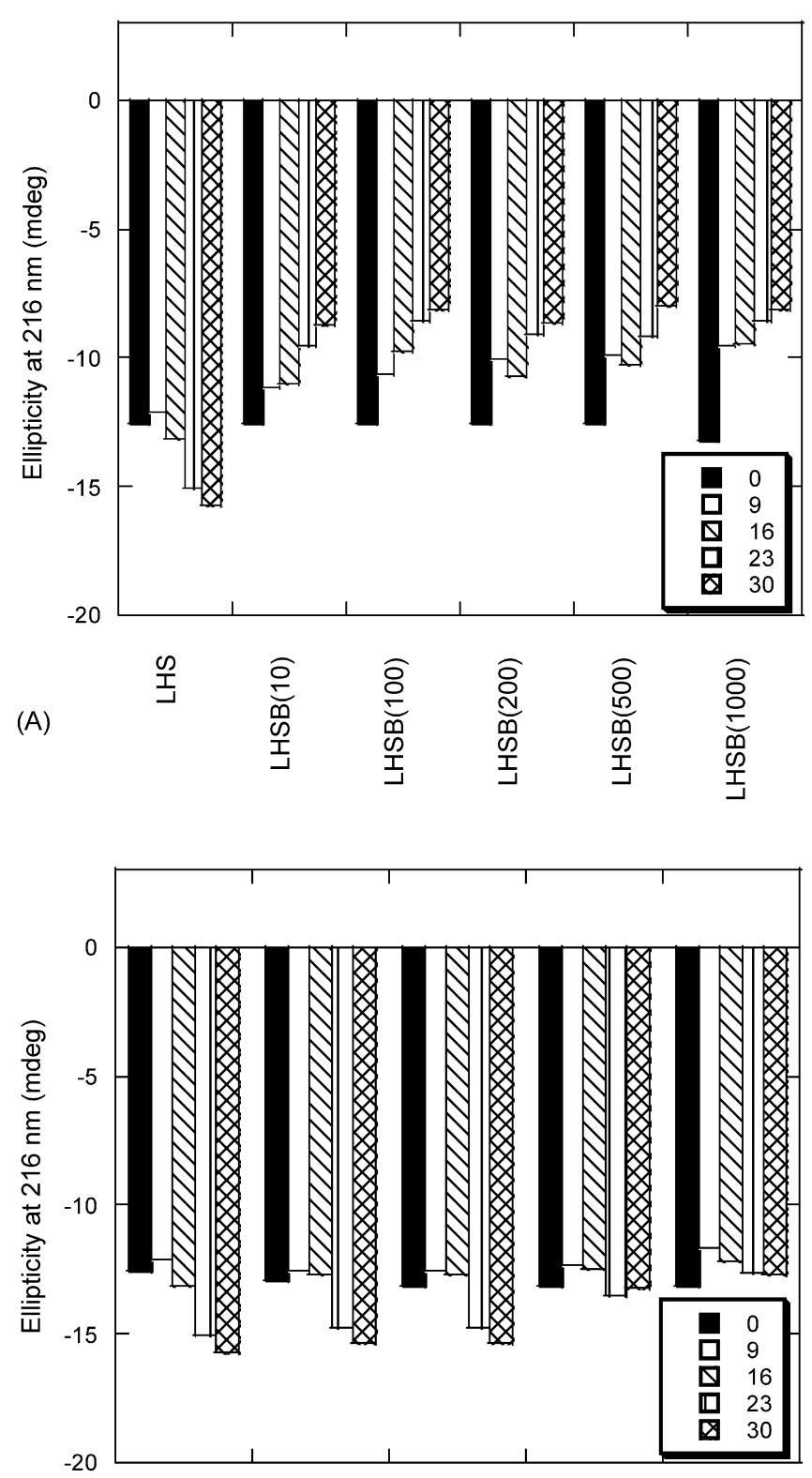

(B)

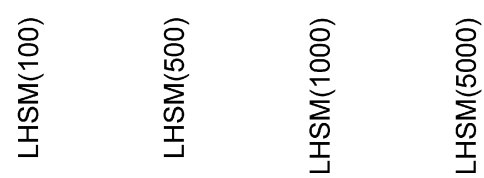

Fig. 9. The effect of dosage of (A) $p$-benzoquinone and (B) melatonin on $\beta$ sheet secondary structure content of hen egg-white lysozyme as monitored by CD ellipticity at $216 \mathrm{~nm}$. Ellipticities at $216 \mathrm{~nm}$ were taken from the CD absorption spectra. Five $(10,100,200,500$ and $1000 \mu \mathrm{M})$ and four $(100,500,1000$ and $5000 \mu \mathrm{M}$ ) different concentration levels are used for $p$-benzoquinone and melatonin, respectively.

on HEWL structural transition, in particular the $\beta$-sheet conformation, were demonstrated by the change of the CD ellipticities at $216 \mathrm{~nm}$. As depicted in Fig. 9A, the ellipticity at $216 \mathrm{~nm}$ of HEWL sample dropped over time at $55^{\circ} \mathrm{C}$, indicating a decrease of $\beta$-sheet secondary structure in the presence of $p$-benzoquinone. In addition, this reduction in $\beta$-conformer content became dramatic with increasing concentration of $p$ benzoquinone from $10 \mu \mathrm{M}$ up to $1000 \mu \mathrm{M}$. However, HEWL sample (pH 2.0) with salt plus 100 or $500 \mu \mathrm{M}$ of melatonin possessed similar secondary structure conformation to that of HEWL (pH 2.0) alone with salt. Melatonin started to exert an inhibitory potency against the formation of $\beta$-sheet structures when the concentration was higher than $500 \mu \mathrm{M}$ (e.g. 1000 and $5000 \mu \mathrm{M})$.

Previous studies demonstrated that $p$-benzoquinone acquired the capability of preventing fibril formation of amyloid proteins/peptides. Examples of such studies include amylin in type II (non-insulin-dependent) diabetes mellitus, $\mathrm{A} \beta$ in Alzheimer's disease [57,58], $\alpha$-synuclein in Parkinson disease [33]. In addition, Tomiyama and co-workers, via the use of immunofluorescence microscopy, proposed that the preventive action of $p$-bezoquinone against amylin fibril-induced toxicity was most likely achieved through the binding between $p$-bezoquinone and amylin aggregated species. These anti-aggregating and antiamyloidogenic potencies against HEWL fibrillar species were also observed in our results.

Melatonin ( $\mathrm{N}$-acetyl-5-methoxytryptamine) is an endogenous neurohormone, produced especially at night in the pineal gland. Its secretion is stimulated by the dark and inhibited by light [3]. It was shown that the melatonin served as a potent anti-oxidant and free radical scavenger [2,47]. Ample evidence indicated that melatonin alleviated the learning and memory deficits in the transgenic mouse model [14], diminished $A \beta$ elicited apoptosis $[15,16,21,49]$.

Apart from these above-stated properties, melatonin was shown to retard the progressive formation of amyloid fibrils and $\beta$-sheets of $A \beta$ species in Alzheimer's disease [26,43,45]. Pappolla et al. concluded that the salt bridges between the side chains of $\mathrm{His}^{+}$and $\mathrm{Asp}^{-}$residues of $\mathrm{A} \beta$, which was suggested to be pivotal to the formation and stabilization of $\beta$-sheet conformation, was disrupted by melatonin [43]. Furthermore, more profound inhibition on $\mathrm{A} \beta$ fibrillogenesis by melatonin was observed in the presence of apoE allele [45]. The two aforementioned studies also proposed that this melatonin-induced inhibition was dependent on structural characteristics rather than on anti-oxidant properties of melatonin [43,45]. In line with the previous results from other studies, our data also revealed that melatonin, at concentrations higher than a certain value, exerted an inhibitory action against the formation of $\beta$-sheet structures in comparison to all other conditions. Evidently, in the range of concentrations employed, $p$-benzoquinone served as a better anti-fibrillogenic/anti-aggregating agent than melatonin. Further research is warranted to examine the underlying mechanism(s) of the interaction between HEWL and melatonin or $p$-benzoquinone.

\section{Conclusions}

At least 20 different disease-related proteins or peptides including $\beta$-amyloid, amylin, $\beta 2$-macroglobulin and $\alpha$ synuclein have been reported to fold abnormally to form amyloid fibrils leading to their accompanying pathologies $[12,24,25,30,48,50,51,59]$. These various proteins and peptides share no obvious similarities in amino acid composition and sequence, yet they are able to self-assemble into fibrils with a 
characteristic cross- $\beta$-sheet structure. In addition, interestingly, accumulating evidence has indicated that proteins unrelated to any amyloidogenic disease aggregate in vitro to form amyloid fibrillar species [17,29,52,53].

Significant numbers of studies suggest that reductions in the inhibition of fibril formation as well as the amount of $\beta$-sheet structure contents are considered as two promising therapeutic approaches in hampering the development of amyloidogenic diseases. A variety of compounds have been reported to retard the formation of aggregated or fibrillar species of amyloid proteins both in vitro and in vivo. For example, evidence showed that various molecules such as alpha-crystallin Hsp20, nicotine, nordihydroguaiaretic acid, rifampicin and others slowed or inhibited fibrillogenesis of $\beta$-amyloid peptides [10,11,31,38-43,56]. Furthermore, several synthesized compounds as well as surfactants [34-36,61] were also found to prevent or delay fibril formation.

In closing, numerous spectroscopic analyses and transmission electron microscopy were used in this work to (1) monitor the HEWL aggregation kinetics with and without salt and (2) assess the anti-amyloidogenic and anti-aggregating abilities of two potential inhibitors, $p$-benzoquinone and melatonin. This study not only seeks for the favorable condition for growing HEWL fibrils but also explores the inhibitory action of two inhibitors on in vitro HEWL fibrillogenesis. Our findings have demonstrated that the formation of large extent of HEWL fibrils was attributed to the addition of salt. We have also shown that $p$-benzoquinone and melatonin exerted a dose-dependent inhibitory activity against $\beta$-sheet formation and aggregation, characteristics of amyloid fibril formation. It is noteworthy to point out that further verification of the role of inhibitors in preventing HEWL fibrillogenesis is still under investigation. Nevertheless, it is our belief that the outcome from this work will enable us, not only to comprehend the mechanism(s) of amyloid protein, disease-related or non-disease-related, self-association process, but also to aid in developing potential targets for molecular therapeutics in the prevention or retardation of amyloid formation implicated in amyloidogenic diseases.

\section{Acknowledgements}

This work was supported by grants from the National Science Council, Taiwan. Authors would like to thank Dr. Rita P.-Y. Chen from Institute of Biochemistry at Academia Sinica, Taiwan for the assistance of CD measurements.

\section{References}

[1] G.H. Beaven, W.B. Gratzer, H.G. Davies, Eur. J. Biochem. 11 (1969) 37

[2] S.M. Beni, R. Kohen, R.J. Reiter, D.X. Tan, E. Shohami, FASEB J. 18 (2004) 149.

[3] A. Brzezinski, N. Engl. J. Med. 336 (1997) 186.

[4] M.J. Burke, M.A. Rougvie, Biochemistry 11 (1972) 2435.

[5] A. Cao, D. Hu, L. Lai, Protein Sci. 13 (2004) 319.

[6] R.W. Carrell, B. Gooptu, Curr. Opin. Struct. Biol. 8 (1998) 799.

[7] R.W. Carrell, D.A. Lomas, Lancet 350 (1997) 134.

[8] R.W. Carrell, D.A. Lomas, Lancet 350 (1997) 134.

[9] F. Chiti, P. Webster, N. Taddei, A. Clark, M. Stefani, G. Ramponi, C.M. Dobson, Proc. Natl. Acad. Sci. U.S.A. 96 (1999) 3590.
[10] F.G. De Felice, M.N. Vieira, L.M. Saraiva, J.D. Figueroa-Villar, J. GarciaAbreu, R. Liu, L. Chang, W.L. Klein, S.T. Ferreira, FASEB J. 18 (2004) 1366.

[11] F.G. Defelice, S.T. Ferreira, An. Acad. Bras. Cienc. 74 (2002) 265.

[12] C.M. Dobson, Semin. Cell Dev. Biol. 15 (2004) 3.

[13] M. Fandrich, V. Forge, K. Buder, M. Kittler, C.M. Dobson, S. Diekmann, Proc. Natl. Acad. Sci. U.S.A. 100 (2003) 15463.

[14] Z. Feng, Y. Chang, Y. Cheng, B.L. Zhang, Z.W. Qu, C. Qin, J.T. Zhang, J. Pineal Res. 37 (2004) 129.

[15] Z. Feng, J.T. Zhang, J. Pineal Res. 37 (2004) 257.

[16] Z. Feng, J.T. Zhang, Free Radic. Biol. Med. 37 (2004) 1790.

[17] A.D. Ferrao-Gonzales, S.O. Souto, J.L. Silva, D. Foguel, Proc. Natl. Acad. Sci. U.S.A. 97 (2000) 6445.

[18] E. Frare, P.P. De Laureto, J. Zurdo, C.M. Dobson, A. Fontana, J. Mol. Biol. 340 (2004) 1153.

[19] S. Fujiwara, F. Matsumoto, Y. Yonezawa, J. Mol. Biol. 331 (2003) 21.

[20] S. Goda, K. Takano, Y. Yamagata, R. Nagata, H. Akutsu, S. Maki, K. Namba, K. Yutani, Protein Sci. 9 (2000) 369.

[21] M.H. Jang, S.B. Jung, M.H. Lee, C.J. Kim, Y.T. Oh, I. Kang, J. Kim, E.H. Kim, Neurosci. Lett. 380 (2005) 26.

[22] S.R. Ji, Y. Wu, S.F. Sui, Gen. Physiol. Biophys. 21 (2002) 415.

[23] Y. Kallberg, M. Gustafsson, B. Persson, J. Thyberg, J. Johansson, J. Biol. Chem. 276 (2001) 12945.

[24] J.W. Kelly, Curr. Opin. Struct. Biol. 8 (1998) 101.

[25] J.W. Kelly, Proc. Natl. Acad. Sci. U.S.A. 95 (1998) 930.

[26] J.E. Kim, M. Lee, Biochem. Biophys. Res. Commun. 303 (2003) 576.

[27] W.E. Klunk, J.W. Pettegrew, D.J. Abraham, J. Histochem. Cytochem. 37 (1989) 1273.

[28] M.R. Krebs, D.K. Wilkins, E.W. Chung, M.C. Pitkeathly, A.K. Chamberlain, J. Zurdo, C.V. Robinson, C.M. Dobson, J. Mol. Biol. 300 (2000) 541.

[29] Z. Lai, W. Colon, J.W. Kelly, Biochemistry 35 (1996) 6470.

[30] P.T. Lansbury Jr., Proc. Natl. Acad. Sci. U.S.A. 96 (1999) 3342.

[31] S. Lee, K. Carson, A. Rice-Ficht, T. Good, Protein Sci. 14 (2005) 593

[32] H. LeVine III, Protein Sci. 2 (1993) 404.

[33] H.T. Li, D.H. Lin, X.Y. Luo, F. Zhang, L.N. Ji, H.N. Du, G.Q. Song, J. Hu, J.W. Zhou, H.Y. Hu, FEBS J. 272 (2005) 3661.

[34] S.J. Lin, Y.J. Shiao, C.W. Chi, L.M. Yang, Bioorg. Med. Chem. Lett. 14 (2004) 1173.

[35] A. Lomakin, D.S. Chung, G.B. Benedek, D.A. Kirschner, D.B. Teplow, Proc. Natl. Acad. Sci. U.S.A. 93 (1996) 1125.

[36] K.J. Marcinowski, H. Shao, E.L. Clancy, M.G. Zagorski, J. Am. Chem. Soc. 120 (1998) 11082

[37] K. Oberg, B.A. Chrunyk, R. Wetzel, A.L. Fink, Biochemistry 33 (1994) 2628.

[38] K. Ono, K. Hasegawa, H. Naiki, M. Yamada, Biochim. Biophys. Acta 1690 (2004) 193.

[39] K. Ono, K. Hasegawa, H. Naiki, M. Yamada, J. Neurosci. Res. 75 (2004) 742 .

[40] K. Ono, K. Hasegawa, M. Yamada, H. Naiki, Biol. Psychiatr. 52 (2002) 880 .

[41] K. Ono, K. Hasegawa, Y. Yoshiike, A. Takashima, M. Yamada, H. Naiki, J. Neurochem. 81 (2002) 434.

[42] K. Ono, Y. Yoshiike, A. Takashima, K. Hasegawa, H. Naiki, M. Yamada, J. Neurochem. 87 (2003) 172.

[43] M. Pappolla, P. Bozner, C. Soto, H. Shao, N.K. Robakis, M. Zagorski, B. Frangione, J. Ghiso, J. Biol. Chem. 273 (1998) 7185.

[44] M.B. Pepys, P.N. Hawkins, D.R. Booth, D.M. Vigushin, G.A. Tennent, A.K. Soutar, N. Totty, O. Nguyen, C.C. Blake, C.J. Terry, et al., Nature 362 (1993) 553.

[45] B. Poeggeler, L. Miravalle, M.G. Zagorski, T. Wisniewski, Y.J. Chyan, Y.B. Zhang, H.Y. Shao, T. Bryant-Thomas, R. Vidal, B. Frangione, J. Ghiso, M.A. Pappolla, Biochemistry 40 (2001) 14995.

[46] V.A. Proctor, F.E. Cunningham, Crit. Rev. Food Sci. Nutr. 26 (1988) 359.

[47] R.J. Reiter, J.J. Garcia, J. Pie, Restor. Neurol. Neurosci. 12 (1998) 135.

[48] C.A. Ross, M.A. Poirier, Nat. Med. 10 (Suppl.) (2004) S10.

[49] Y.X. Shen, S.Y. Xu, W. Wei, X.L. Wang, H. Wang, X. Sun, J. Pineal Res. 32 (2002) 163.

[50] J.D. Sipe, Annu. Rev. Biochem. 61 (1992) 947. 
[51] J.D. Sipe, Crit. Rev. Clin. Lab. Sci. 31 (1994) 325.

[52] V. Sluzky, A.M. Klibanov, R. Langer, Biotechnol. Bioeng. 40 (1992) 895.

[53] V. Sluzky, J.A. Tamada, A.M. Klibanov, R. Langer, Proc. Natl. Acad. Sci. U.S.A. 88 (1991) 9377.

[54] C. Soto, J. Mol. Med. 77 (1999) 412.

[55] J.P. Taylor, J. Hardy, K.H. Fischbeck, Science 296 (2002) 1991.

[56] T. Thomas, T.G. Nadackal, K. Thomas, Neuroreport 12 (2001) 3263.

[57] T. Tomiyama, H. Kaneko, K. Kataoka, S. Asano, N. Endo, Biochem. J. 322 (Pt. 3) (1997) 859.
[58] T. Tomiyama, A. Shoji, K. Kataoka, Y. Suwa, S. Asano, H. Kaneko, N. Endo, J. Biol. Chem. 271 (1996) 6839.

[59] V.N. Uversky, A.L. Fink, Biochim. Biophys. Acta 1698 (2004) 131.

[60] M.C. Vaney, S. Maignan, M. Ries-Kautt, A. Ducriux, Acta Crystallogr. D: Biol. Crystallogr. 52 (1996) 505.

[61] S.S. Wang, Y.T. Chen, S.W. Chou, Biochim. Biophys. Acta 1741 (2005) 307.

[62] S.S. Wang, T.A. Good, D.L. Rymer, Protein Sci. 14 (2005) 1419. 